\title{
Magnetorquer Based Attitude Control for a Nanosatellite Testplatform
}

\author{
Daniel M. Torczynski* and Rouzbeh Amini ${ }^{\dagger}$ \\ Delft University of Technology, Delft, 2629 GZ, The Netherlands \\ Paolo Massioni $\ddagger$ \\ MicroNed, Delft, 2628 GZ, The Netherlands
}

\begin{abstract}
This paper presents the design of an attitude controller for a magnetically actuated nanosatellite. The goal of this attitude control system is to be able to dump excess kinetic rotational energy as well as to point to a rotating frame with an accuracy of five degrees on each axis. From the options considered, a locally asymptotically stable PD type controller is chosen as the best solution to the local attitude control pointing problem. This design choice is verified via simulation which includes actuator constraints, environmental disturbance torques due to drag, solar radiation, residual dipoles and gravity, and satellite models from the cubesat Delfi-n3Xt.
\end{abstract}

\section{Nomenclature}

$\underline{1}_{n \times n}$ Identity Matrix (nxn)

$\mu \quad$ Earth's Gravitational Constant

$\Phi \quad$ State Transition Matrix

$\Psi \quad$ Monodromy Matrix

$\omega_{0} \quad$ Orbital Period

$\omega_{a b}^{c} \quad$ Angular rate between frame a and b expressed in frame c

$b \quad$ Earth's Magnetic Field

I Inertia Matrix

$m \quad$ Magnetic Dipole Moment

${ }_{b}^{a} q \quad$ Quaternion from reference frame $\mathrm{b}$ to frame a

$\hat{R}_{c m}^{c}$ Distance from Earth's center to satellite's center of gravity

$T^{c} \quad$ Torque in frame c

$\underline{S}($ ) Skew Symmetric Cross Matrix

\section{Introduction}

The purpose of this paper is to evaluated the performance of several types of attitude controllers for a cubesat using only magnetorquer-based actuation. As satellite miniaturization progresses to the cubesat level(satellite structure is $10 \mathrm{~cm} \times 10 \mathrm{~cm} \times 34 \mathrm{~cm}$ ), attitude controllers must be designed to operate for this new class size. Actuator miniaturization, however, lags most other bus hardware systems, and the full complement of actuators available for commercial-sized satellites is not yet available to cubesats. Prototypes of miniaturized reaction wheels and thrusters will be flown as experimental payloads on Delfi-n3Xt, leaving only magnetorquers as a viable option for active attitude control. In addition, in future missions once thrusters and reaction wheels have been properly space certified, magnetorquer-based attitude control will be used as a backup system, as magnetorquers will still be onboard for use in rate damping and momentum

\footnotetext{
*MSc Student, Space Systems Engineering, Kluyerweg 12629 HS Delft, Student Member.

${ }^{\dagger}$ Ph.D Candidate, Space Systems Engineering, Kluyerweg 12629 HS Delft, Student Member.

${ }^{\ddagger}$ Ph.D Candidate, Delft Center for Systems and Control, Mekelweg 22628 CD Delft, Student Member.
} 
dumping. Consequently, the goal of this paper is to determine the best magnetorquer-based attitude control system for Delfi-n3Xt, given the restricted power budget available to a three-unit cubesat. Mission success requires the ability to track a rotating reference frame. Rotating reference frame tracking is required to test an experimental thruster, in order to point the thrust vector in the direction of orbital velocity to an accuracy of five degrees on the velocity axis. While there is no fixed requirement for settling time, prior magnetorquer performance of a cubesat has suggested on the order of 1-2 orbits ${ }^{1,2}$ starting from a $30^{\circ}$ error on each axis. While this is in part due to power restrictions, it is largely due to the unavoidable fundamental problem of underactuation when dealing with magnetorquers. In an approximately polar low Earth orbit, the magnetic field rotates twice per orbit, meaning a previously uncontrollable axis now becomes fully controllable within a quarter orbit later. This suggests a theoretical limit of approximately 25 minutes ( $1 / 4$ orbit) for settling time to any given random initial attitude, a limit which is considered ideal during the design of this attitude controller.

The field of magnetorquer-based attitude control has been extensively covered over the past decades. One approach is to use a classical PD type control which assumes three independent torques can be applied to the spacecraft. ${ }^{3}$ That design develops both a model dependent and independent controller, both of which are proven to be asymptotically stable about a zero error equilibrium. Another PD type controller is shown to be globally asymptotically stable by employing a time varying proportional gain with the aid of gravity gradient stability. ${ }^{4}$ An alternate control approach is an LQR-based solution which can be either time varying or time invariant. ${ }^{5}$ This approach takes advantage of the quasi-periodicity of the Earth's magnetic field, and allows for Floquet stability analysis to be performed after the fact. The LQR solution can be modified by adding integrators in order to counteract steady-state disturbance torques. Furthermore, this solution can use the infinite gain margin property of LQR control to employ saturation logic in order to maintain controller stability. ${ }^{6}$ Several cubesat missions have also explored using an LQR controller including AAUSAT I, Compass I, and Swisscube. In addition to LQR, another potential optimal approach is model predictive control (MPC). Using a magnetic field model, a closed form solution can be derived which includes actuator constraints. ${ }^{7}$ In addition, an eMPC for a magnetically actuated satellite can be shown to maintain accuracy while achieving considerable decreases in energy consumption. ${ }^{8}$ Because it depends strongly on the magnetic field model, MPC performance can vary significantly when using a constant magnetic field assumption versus a time varying magnetic field model. ${ }^{9}$ This analysis finds signficant acceleration and angular rate reduction when using the time varying model. Moreover, it shows that the addition of a terminal penalty has a stabilizing effect on the control law.

In this paper we present the modeling and implementation of the detumbling and local attitude control system for the cubesat Delfi-n3Xt. In Section II, the spacecraft dynamics model is developed which is used later in Section III to design the detumbling and pointing controllers. Section IV presents the simulations of the implementation these two controllers on Delfi-n3Xt subject to actuator constraints and disturbance torques. Section V summarizes the controllers' performance and gives arguments for the final attitude controller design choice.

\section{Spacecraft Model}

\section{A. Reference Frames}

For the setup of the attitude control problem, the following reference systems are adopted:

- Inertial Axes $\left(X_{i} Y_{i} Z_{i}\right)$ : The origin of this frame is at the Earth's center of mass. The $X$ axis points toward the vernal equinox and $Z$ axis points to the geographic north pole.

- Control Axes $\left(X_{c} Y_{c} Z_{c}\right)$ : The origin of this frame is at the satellite's center of mass. The axes are aligned with the spacecraft principle moments of inertia. It is assumed that the body axes and control axes are aligned.

- Orbital Axes $\left(X_{o} Y_{o} Z_{o}\right)$ : The origin of this frame is at the satellite's center of mass. The $X$ axis is along the orbit normal, $Z$ axis is in the zenith direction. 


\section{B. Kinematics}

One of the most common parameterizations of attitude is given by the four Euler parameters. Using these variables, the following representation for attitude kinematics can be derived: ${ }^{10}$

$$
\dot{q}=\frac{1}{2} \underline{\Omega} q \quad \text { where } \quad \underline{\Omega}=\left[\begin{array}{rrrr}
0 & \omega_{z} & -\omega_{y} & \omega_{x} \\
-\omega_{z} & 0 & \omega_{x} & \omega_{y} \\
\omega_{y} & -\omega_{x} & 0 & \omega_{z} \\
-\omega_{x} & -\omega_{y} & -\omega_{z} & 0
\end{array}\right]
$$

where $q \in \mathbf{R}^{4}$ is the unit norm vector of Euler's parameters and $\omega \in \mathbf{R}^{3}$ is the spacecraft angular rate. The choice of quaternion for attitude parameterization is motivated by its property of nonsingular representation.

\section{Dynamics}

The attitude dynamics of a rigid body can be expressed by the following equation: ${ }^{10}$

$$
I \dot{\omega}^{c}=-\omega^{c} \times I \omega^{c}+T_{c t r l}^{c}+T_{d}^{c}
$$

where $I \in \mathbf{R}^{3}$ is the inertia matrix in the control reference frame, $T_{c t r l}^{c} \in \mathbf{R}^{3}$ is the vector of magnetic control torques, and $T_{d}^{c} \in \mathbf{R}^{3}$ is the vector of external disturbance torques. The control torque is given by:

$$
T_{c t r l}^{c}=m^{c} \times b^{c}
$$

where $m^{c} \in \mathbf{R}^{3}$ is the magnetorquer produced magnetic dipole moment and $b^{c} \in \mathbf{R}^{3}$ is the Earth's magnetic field in Tesla. The disturbance torque is given by:

$$
T_{d}^{c}=T_{d r a g}^{c}+T_{r a d}^{c}+T_{m a g}^{c}+T_{g g}^{c}
$$

where $T_{d r a g}^{c}, T_{\text {rad }}^{c}, T_{m a g}^{c}$, and $T_{g g}^{c}$ are the disturbance torques due to atmospheric drag, radiation pressure, residual magnetic dipoles, and gravity gradient respectively.

\section{Linearization}

From the nonlinear models given by Eq. (1)-Eq. (4), a linearized system is derived for later use in linear control theory. The nonlinear system takes on the form $\dot{x}=f(x, u)$ where the state vector, $x$, is defined as $x=\left[\begin{array}{cc}\bar{q} & \bar{\omega}\end{array}\right]$ and the control signal, $u$, is defined as $u=m^{c}$. The fourth term of the quaternion can be left out of the state vector due to its relationship to the other terms given by $q_{1}^{2}+q_{2}^{2}+q_{3}^{2}+q_{4}^{2}=1$. This system is linearized about a trajectory given by the rotating reference frame defined as:

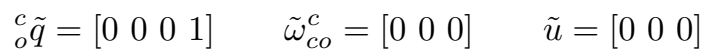

For this case these states express the nominal rotation from the orbital to the control reference frame, the nominal angular rate between these two reference frames, and the nominal control input. The nonlinear system can now be written:

$$
\dot{x}=\mathrm{A} x+\mathrm{B} u
$$

where the system matrices $\mathrm{A}$ and $\mathrm{B}$ are given as:

$$
\mathrm{A}=\left.\frac{\partial f(x, u)}{\partial x}\right|_{x=\tilde{x}} \quad \mathrm{~B}=\left.\frac{\partial f(x, u)}{\partial u}\right|_{u=\tilde{u}}
$$

For orbital reference frame tracking, the $\omega$ given in Eq. (1) is a rotation rate from the orbital to the control reference frame, $\omega_{c o}^{c}$, where as the $\omega$ given in Eq. (2) is a rotation rate from the inertial frame to the control frame or $\omega_{c i}^{c}$. In order to combine these into one linear model the following relationship between the two rotation rates must be used:

$$
\omega_{c o}^{c}=\omega_{c i}^{c}-R_{o}^{c} \omega_{o i}^{o} \quad \text { where } \quad \omega_{o i}^{o}=\left[\begin{array}{lll}
\omega_{0} & 0 & 0
\end{array}\right]
$$


where $R_{o}^{c}$ is a rotation matrix from the orbital to control reference frame. In addition, due to its ease of linearization in the orbital reference frame, the linearized gravity gradient torque is also included in the system model: ${ }^{5}$

$$
T_{g g}^{c}=\frac{3 \mu}{R_{c m}^{3}}\left(\hat{R}_{c m}^{c} \times I \hat{R}_{c m}^{c}\right) \quad \approx \quad 6 \omega_{0}^{2}\left[\begin{array}{c}
\left(I_{z}-I_{y}\right) \delta q_{1} \\
\left(I_{z}-I_{x}\right) \delta q_{2} \\
0
\end{array}\right]
$$

where $\delta q=\left[\begin{array}{llll}\delta q_{1} & \delta q_{2} & \delta q_{3} & 1\end{array}\right]$ and is a small deviation of the attitude quaternion, ${ }_{o}^{c} q$, from the nominal. An additional operation has been applied to the original magnetic dipole moment, $m$, to create a more efficient moment $\tilde{m}$. This operation maps the original, ideal moment, to the plane perpendicular to the magnetic field line, thereby removing the component of the moment vector which contributes no torque: ${ }^{11}$

$$
T_{c t r l}^{c}=\tilde{m}^{c} \times b^{c} \quad \text { where } \quad \tilde{m}^{c}=\frac{m^{c} \times b^{c}}{\|b\|}
$$

Given the above linearization procedure described in Eq. (5) - Eq. (10), the state vector $x$ and corresponding system matrices A and B are given as:

$$
x=\left[\begin{array}{c}
\delta q_{1} \\
\delta q_{2} \\
\delta q_{3} \\
\delta \omega_{x} \\
\delta \omega_{y} \\
\delta \omega_{z}
\end{array}\right] \quad \mathrm{A}=\left[\begin{array}{cc}
-\underline{S}(\tilde{\omega}) & \underline{1}^{2} \underline{1}_{3 \times 3} \\
\underline{T}_{g g 3 \times 3} & \underline{\mathrm{I}}^{-1}[\underline{S}(\underline{\mathrm{I}} \omega)-\underline{S}(\tilde{\omega}) \underline{\mathrm{I}}]
\end{array}\right] \quad \mathrm{B}=\left[\begin{array}{c}
\underline{0}_{3 \times 3} \\
\left.\left.\underline{\mathrm{I}}^{-1} \frac{1}{\|b\|}\left[\underline{S}^{o} b\right)\right]\left[\underline{S}{ }^{o} b\right)\right]
\end{array}\right]
$$

where $\delta \omega$ is a small deviation of the angular velocity, $\omega_{c o}^{c}$, from the nominal, and $\underline{T}_{g g}{ }_{3 \times 3}$ is $\underline{0}_{3 \times 3}$ with $T_{g g}$ on the diagonals.

\section{Control Design}

\section{A. Detumbling Controller}

Upon exiting the orbital deployer, the cubesat will experience some of the highest rotation rates it will see during its mission lifetime. Estimated maximum rates are $10 \frac{\mathrm{deg}}{\mathrm{s}}$ on each axis. In order to dump this excess energy, a detumbling controller will be used to bring body rotational rates down to below $1 \frac{\mathrm{deg}}{\mathrm{s}}$ on each axis before the attitude controller will become operational. This detumbling controller takes the form of the well-known b-dot controller: ${ }^{2}$

$$
m=-C \dot{b}
$$

where $C$ is a positive definite gain matrix. Under the assumption that the magnetic field vector's change results from spacecraft rotation, $\dot{b} \approx b \times \omega$, this controller effectively applies torque opposite the direction of rotation, thereby reducing the satellite's kinetic energy.

Due to noisy sensor readings of the magnetic field, differentiation of the magnetic field can be problematic. It is common practice to use a first-order state variable filter to estimate the time derivative of $b$ :

$$
H(s)=\frac{\hat{b}}{b}(s)=\frac{s \omega_{c}}{s+\omega_{c}}
$$

where $\hat{b}$ is the estimate of the time derivative of $b$. For high frequency inputs $\left(s>>\omega_{c}\right)$ this tranfer function behaves like a simple gain, and for low frequency inputs $\left(s<<\omega_{c}\right)$ the function is a differentiator in approximation. In this way $\omega_{c}$ can be used as a filtering cutoff frequency.

\section{B. Linear Quadratic Regulator}

\section{Problem Formulation}

A constant gain linear quadratic regulator (LQR) is developed as a stablizing control law for a linear timeinvariant system. From prior research, the constant gain LQR has been found to be comparable in performance to an infinite horizon and finite horizon controller. ${ }^{5}$ For this reason the constant gain controller was 
chosen as the test case LQR due to its lowest computational load. The problem is formulated as finding a full-state feedback control law minimizing the infinite time linear quadratic cost function:

$$
J(u)=\int_{0}^{\infty}\left[x^{T}(t) Q x(t)+u^{T}(t) R u(t)\right] d t
$$

where the weighting matrices $Q$ and $R$ are positive semidefinite and positive definite respectively. The above cost function is also subject to the linearized state dynamic equations given by Eq. (6) with a slight modification on B:

$$
\dot{x}(t)=\mathrm{A} x(t)+\bar{B} u(t) \quad \text { and } \quad x(0)=x_{0}
$$

where $\bar{B}$ is the control matrix $\mathrm{B}$ averaged over one time period $(\mathrm{T})$ :

$$
\bar{B}=\frac{1}{T} \int_{t_{0}}^{t_{0}+T} \mathrm{~B}(t) d(t)
$$

Because the system is linear, time-invariant and controllable, a control law can be implemented from the solution to the steady state Riccati equation. ${ }^{13}$ This optimal law is given by:

$$
u=R^{-1} \bar{B}^{T} P x \quad \text { or equivalently } \quad m^{c}=K x
$$

where $P$ satisfies the Riccati Algebraic Equation:

$$
P^{T} A+P A-P \bar{B} R^{-1} B^{T} P+Q=0
$$

\section{Stability}

Stability of the LQR approach must be verified because although constant gain LQR theory guarantees closed loop stability for linear time-invariant systems, it does not do so for periodic linear systems. In order to check whether the system is stable for a given $Q$ and $R$, Floquet analysis is employed. ${ }^{14}$ This analysis states that a periodic linear system is asymptotically stable only if its complex characteristic multipliers lie on the open unit disk. The characteristic multipliers are calculated as the eigenvalues of the monodromy matrix, given by:

$$
\Psi\left(t_{0}\right)=\int_{t_{0}}^{T+t_{0}} \tilde{\mathrm{A}} \Phi\left(t, t_{0}\right) d t \quad \text { where } \quad \tilde{\mathrm{A}}=\mathrm{A}-\mathrm{B}(t) K
$$

The constant $T$ is the orbital period and $\Phi$ is the state transition matrix from reference time $t_{0}$. In addition, the weight matrices $Q$ and $R$ can be tuned from this analysis. The matrix $Q$ can be set as a function of a scalar tuning variable $p:^{2}$

$$
Q=\left[\begin{array}{cc}
p k_{1} \underline{1} & \underline{0} \\
\underline{0} & p k_{2} \underline{1}
\end{array}\right]^{T}\left[\begin{array}{cc}
\frac{1}{2} \underline{1} & \frac{1}{2} \underline{1} \\
\frac{1}{2} \underline{1} & \frac{1}{2} \underline{1}
\end{array}\right]\left[\begin{array}{cc}
p k_{1} \underline{1} & \underline{0} \\
\underline{0} & p k_{2} \underline{1}
\end{array}\right]
$$

where $\underline{0}$ and $\underline{1}$ are both $3 \times 3$ matrices, $k_{1}=0.001$ and $k_{2}=0.00001$. The variable $p$ can then be swept through a range of values and the maximum absolute value of the characteristic multipliers plotted as a function of $p$. The $p$ which minimizes the absolute value of the maximum eigenvalue of the monodromy matrix corresponds to the weighting which generates the fastest system response time, and this $Q$ can be employed as the optimal state weighting.

\section{PD Controller}

A PD controller is tested due to its advantage as one of the least computationally intensive control approaches. One type of PD controller is that which includes both a rate damping term, $K_{D}$, and a quaternion term, $K_{P}$, as given below: ${ }^{4}$

$$
m^{c}(t)=K_{D} \omega_{c o}^{c} \times b^{c}+K_{P}{ }_{o}^{c} q \times b^{c}
$$

Plugging this $m^{c}$ into Eq. (6) for $u$ yields the new linearized form given by:

$$
\dot{x}=\mathrm{A} x+\mathrm{B}\left(K_{P} \delta q+K_{D} \delta \omega\right)
$$

$$
5 \text { of } 9
$$


Table 2. Simulation Parameters

Table 1. Algorithm Parameters

\begin{tabular}{|c|c|}
\hline Parameter & Value \\
\hline$C$ & 11000 \\
\hline$w_{c}$ & 2 \\
\hline$p$ & 26 \\
\hline$R$ & $\underline{1}_{3 \times 3}$ \\
\hline$K_{P}$ & $\operatorname{diag}(-0.03 \quad-0.03 \quad-0.15)$ \\
\hline$K_{D}$ & $\operatorname{diag}(-21 \quad-21 \quad-35)$ \\
\hline
\end{tabular}

\begin{tabular}{c||c||c}
\hline Parameter & Value & Unit \\
\hline \hline Inertia Matrix & $\operatorname{diag}(0.0370 .0360 .006)$ & {$\left[\mathrm{kgm}^{2}\right]$} \\
Max. Magnetic Moment & 0.06 & {$\left[\mathrm{Am}^{2}\right]$} \\
Accuracy Required & {$\left[\begin{array}{ll}5 & 5\end{array}\right]$} & {$[\mathrm{deg}]$} \\
Orbital Angular Velocity & $1.079 \times 10^{-3}$ & {$[\mathrm{rad} / \mathrm{s}]$} \\
Initial Attitude & {$\left[\begin{array}{lll}3030 & 30\end{array}\right]$} & {$[\mathrm{deg}]$} \\
Initial Angular Velocity & {$\left[\begin{array}{lll}1 & 1 & 1\end{array}\right] 10^{-3}$} & {$[\mathrm{rad} / \mathrm{s}]$} \\
Atmospheric Density & $1.68 \times 10^{-14}$ & {$\left[\mathrm{~kg} / \mathrm{m}^{3}\right]$} \\
Residual Dipole Moment & {$\left[\begin{array}{lll}0 & 0.001\end{array}\right]$} & {$\left[\mathrm{Am}^{2}\right]$} \\
\hline
\end{tabular}

Equation(22) can be rewritten in the form:

$$
\dot{x}=\hat{\mathrm{A}} x \quad \text { where } \quad \hat{\mathrm{A}}=\mathrm{A}+\mathrm{B}\left[\begin{array}{ll}
K_{P} & K_{D}
\end{array}\right]
$$

Now Floquet analysis can be employed by setting $\hat{\mathrm{A}}=\tilde{\mathrm{A}}$ from Eq. (19). In addition the values in $K_{P}$ and $K_{D}$ can be varied much like the parameter $\mathrm{p}$ in order to find an optimal gain matrix.

\section{Simulation}

In this section the simulations are presented. Table 1 outlines the parameters used by the detumbling, LQR, and PD controllers. These weightings were computed by the methods described in the previous section on control design, and are used to calculate the gains used in these controllers. Table 2 displays the parameters used in the simulation. The moments of inertia, maximum dipole moments, and residual dipole moment are taken from the latest Delfi-n3Xt cubesat designs. ${ }^{15}$ The simulation uses an IGRF 2010 model to compute the Earth's magnetic field as a function of position. The results of the simulation can be seen in the figures below.

The result of the detumbling simulation is within normal expectations, as seen in Fig. 1. This simulation included actuator saturation and all disturbance torques. The satellite started from a $10 \frac{\mathrm{deg}}{\mathrm{s}}$ rotation rate on each axis and settled to roughly $0.2 \frac{\mathrm{deg}}{\mathrm{s}}$ after 10000 seconds or just under 2 orbits. This steady state rate was used as the initial rotation rate for all the pointing simulations. Fig. 2 shows the stability analysis performed for the LQR controller. The optimal $Q$ occurs at $p=17$, which was proven in performance by linear simulations without disturbance torques. However, in the nonlinear simulation that included disturbance torques, it was found that a non-optimal tuning parameter of 26 was more effective at rejecting those disturbances. Ultimately, the LQR controller was found to be inflexible in terms of gain scaling, simply due to the boundaries imposed by stability requirements of having a maximum absolute eigenvalue less than

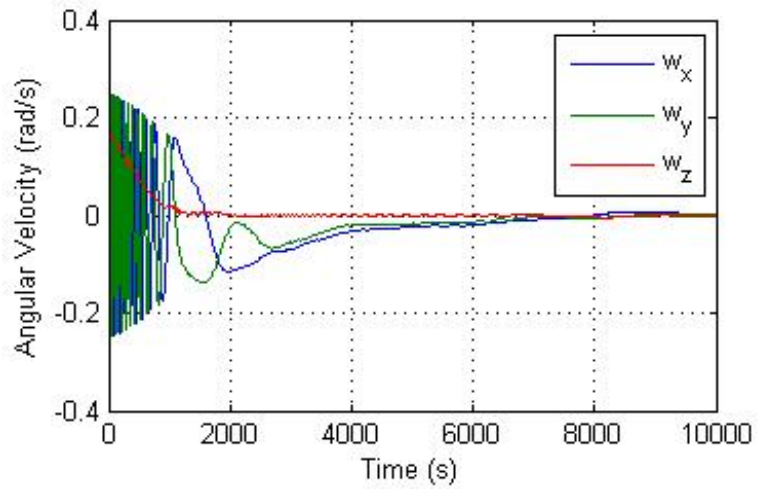

Figure 1. Detumbling controller

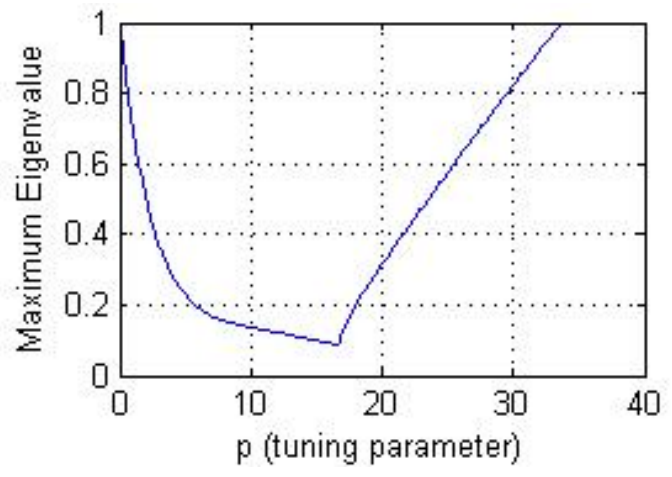

Figure 2. $Q$ matrix tuning 


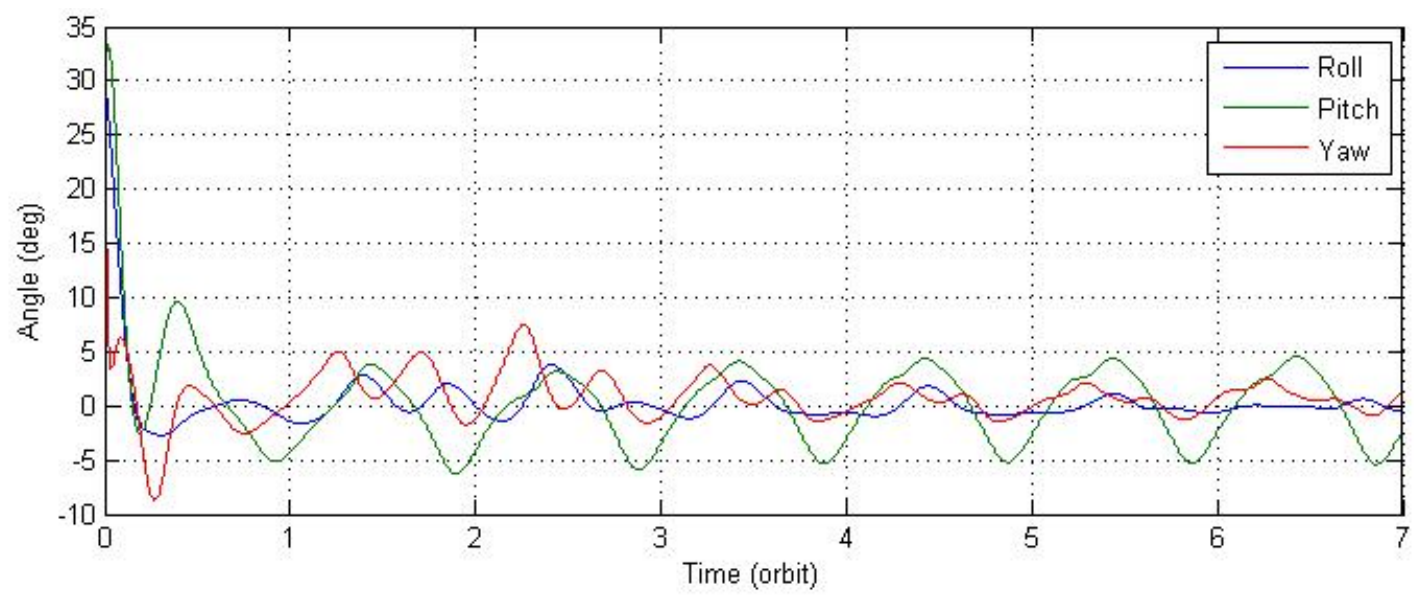

Figure 3. LQR control law pointing errors including disturbance torques.

one. Changing the control matrix $R$ was ineffective because it is the relationship between $Q$ and $R$ and not the absolute value that determines the control gains. The PD controller had more flexibility in gain scaling, as both relative and absolute values between the $K_{P}$ and $K_{D}$ matrices are important. In this way the gains were increase while maintaining Floquet stability in order to better reject disturbance torques. However, it was found that after a certain point, increasing $K_{P}$ and $K_{D}$ did not improve performance, but merely consumed more power. The figures below show the orbital frame tracking results and Table 3 summarizes key findings from these simulations. Both controllers have $5^{\circ}$ settling times of roughly $\frac{1}{2}$ an orbit. The PD controller, however, is $23 \%$ faster or almost 700 seconds. From the same plots, it can also be seen that the PD controller has better steady state performance than the LQR controller. The PD controller has a maximum steady state error of about $2^{\circ}$.

The LQR controller, on the other hand, has much larger pitch errors at almost $5.5^{\circ}$. Both of these steady state errors are periodic due to the periodic nature of the radiation pressure and residual dipole moment disturbance torques. This increase in performance comes at a higher energy cost as seen in Fig. 5. The energy consumption is artificially divided into two time segments for analysis. The first segment is the energy required to achieve the steady state, which in Table 3 is labeled as 3000s Energy, or energy required in the first 3000 seconds. The LQR controller performs slightly better consuming only $86 \%$ of what the PD controller consumes.

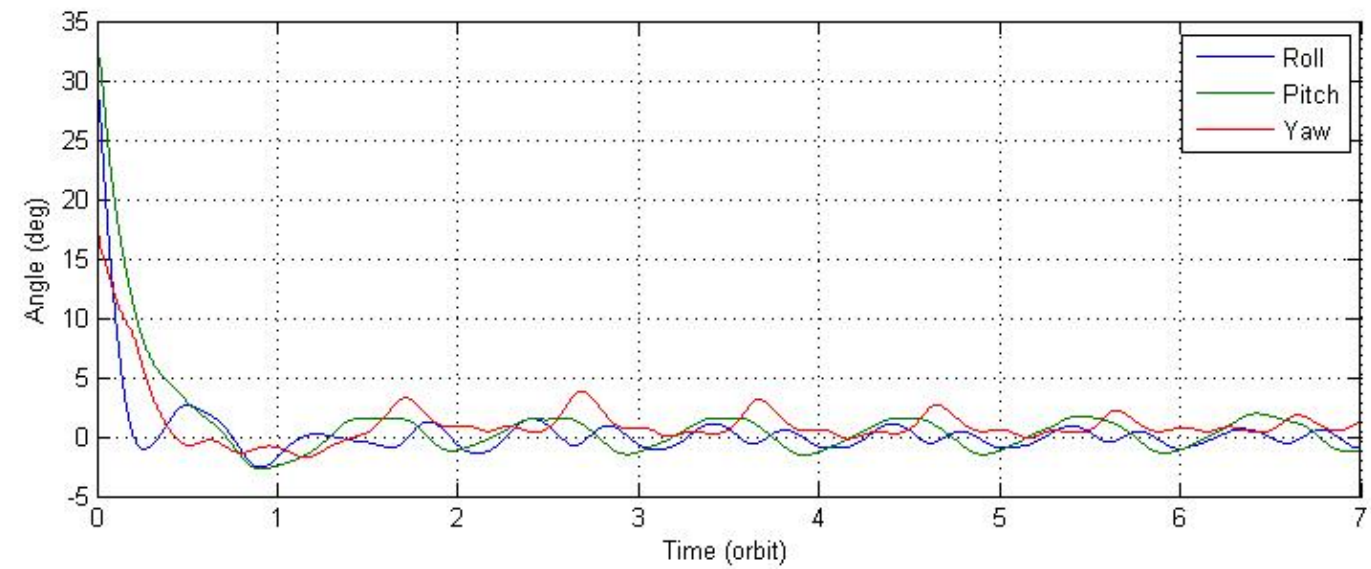

Figure 4. PD control law pointing errors including disturbance torques. 


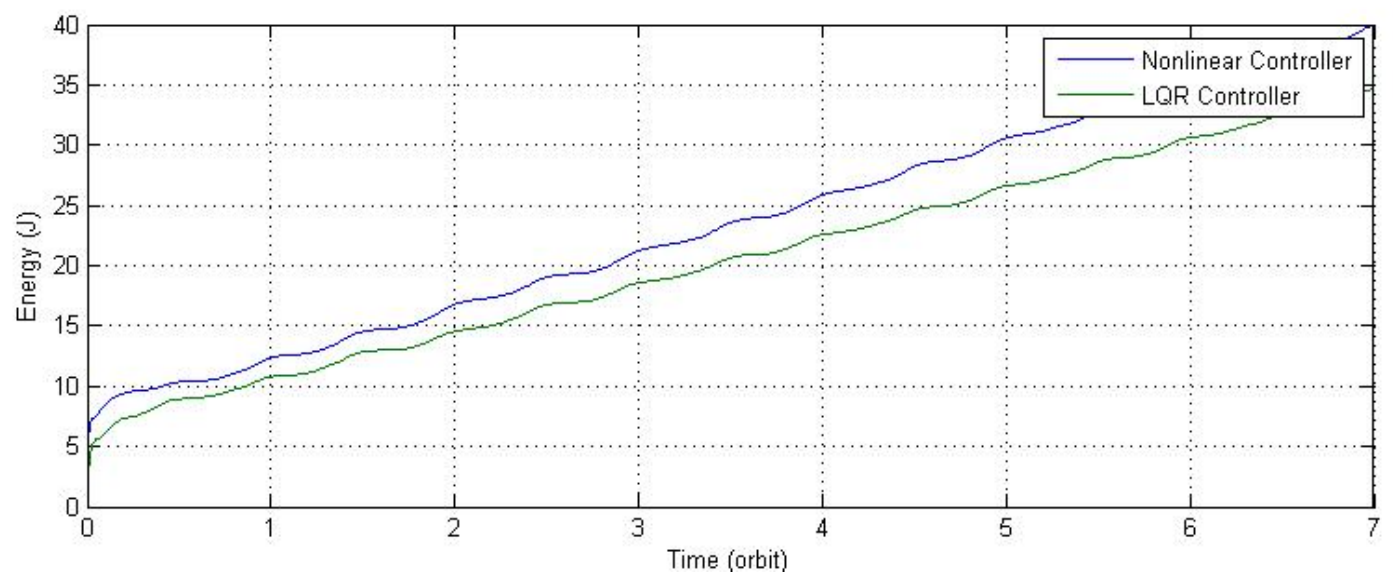

Figure 5. Total energy consumed by LQR and PD control laws

Table 3. Simulation Summary

\begin{tabular}{c||c|c}
\hline Parameter & LQR Value & PD Value \\
\hline \hline $5^{\circ}$ Settling Time [s] & 3077 & 2385 \\
Max Steady State Error [deg] & 5.5 & 2 \\
3000s Energy [J] & 8.96 & 10.42 \\
Avg Steady State Power [mW] & 1.5 & 1.6
\end{tabular}

\section{Conclusions}

The second segment is the average power required to maintain steady state, which is calculated as the average power consumption from 3000s to the end of the simulation. In this criteria the LQR controller again performs slightly better, using only $94 \%$ of the power required by the PD controller. The PD controller was selected as the tracking controller for the Delfi-n3Xt satellite attitude control system. This choice is motivated by its superior performance, even at the expense of larger power requirements. The difference in settling times and steady state errors at $25 \%$ and $65 \%$ respectively is significant. In addition, one factor not considered in this paper, but which is also a contributing factor to reference frame tracking error is the attitude determination error due to sensor bias and noise. For Delfi-n3Xt, both attitude determination and attitude control were given their own accuracy requirements in order to achieve a total ADCS pointing accuracy. While both controllers meet the requirements for control pointing accuracy, the additional PD performance improvement beyond the required accuracy can be used to augment determination performance in order to achieve total ADCS pointing accuracy. The difference in power consumption is not considered significant enough to be a determining factor in controller choice. While the LQR controller uses $14 \%$ less to reach the steady state, in practice this translates to the magnetorquers being saturated for about 20 more seconds in the beginning for the PD controller than the LQR controller. The Delfi-n3xt power budget can accomodate magnetorquer power saturation for several hours, so a difference of 20 seconds is trivial. Similarly, a difference in steady state consumption of less than $1 \mathrm{~mW}$ is also trivial.

\section{Acknowledgments}

The authors thank the Delfi-n3Xt cubesat team for their continued support and encouragement. The authors also thank the U.S. Fulbright Program for its support of independent research projects across the world, and in particular, the grant which supported this research. 


\section{References}

${ }^{1}$ Giebelmann, J., "Development of an Active Magnetic Attitude Determination and Control System for Picosatellites on Highly Inclined Circular Low Earth Orbits," Master's Thesis, Department of Aerospace Engineering, Royal Melbourne Institute of Technology, Melbourne, Australia, 2006.

${ }^{2}$ Graversen, T., Frederiksen, M.K., and Vedstesen, S.V., "Attitude Control System for AAU CubeSat," Master's Thesis, Department of Control Engineering, Aalborg University, Aalborg, Denmark, 2002.

${ }^{3}$ Wen, J.T.-Y., and Kreutz-Delgado, K., "The Attitude Control Problem," IEEE Transactions on Automatic Control, Vol. 36, No. 10, Oct. 1991, pp. 1148-1162.

${ }^{4}$ Wiśniewski, R., and Blanke, M., "Fully Magnetic Attitude Control for Spacecraft Subject to Gravity Gradient," Automatica, Vol. 35, No. 7, Jul 1999, pp. 1201-1214.

${ }^{5}$ Wiśniewski, R., "Satellite Attitude Control Using Only Electro-magnetic Actuation," Ph.D Thesis, Department of Control Engineering, Aalborg University, Aalborg, Denmark, 1996.

${ }^{6}$ Psiaki, M., "Magnetic Torquer Attitude Control via Asymptotic Periodic Linear Quadratic Regulation," Journal of Guidance, Control, and Dynamics, Vol. 23, No. 3, May-June 2000, pp.532-538.

${ }^{7}$ Silani, E., and Lovera, M., "Magnetic Spacecraft Attitude Control: a Survey and Some New Results," Control Engineering Practice, Vol. 13, No. 3, Mar 2005, pp. 357-371.

${ }^{8}$ Krogstad, T., Gravdahl, J., and Tondel, P., "Explicit Model Predictive Control of a Satellite with Magnetic Torquers," Proceedings of the 2005 IEEE International Symposium on, Mediterranean Conference on Control and Automation, IEEE, Limassol, Cyprus, 2005, pp. 491-496.

${ }^{9}$ Wood, M., Chen, W.-H., Fertin, D., "Model Predictive Control of Low Earth Orbiting Spacecraft with Magnetotorquers," Proceedings of the 2006 IEEE International Conference on Control Applications, IEEE, Munich, Germany, 2006, pp. 2908-2913.

${ }^{10}$ Wertz, J., Spacecraft Attitude Determination and Control, D. Reidel, Dordrecht, 1978.

${ }^{11}$ Martel, F., Pal, P.K., and Psiaki, M.L., "Active Magnetic Control System for Gravity Gradient Stabilized Spacecraft," Proceedings of the 1988 Annual AIAA/USU Conference on Small Satellites, AIAA, Logan, Utah, 1988, pp. unnumbered.

${ }^{12}$ Stickler, A., and Alfriend, K., "An Elementary Magnetic Attitude Control System," Journal of Spacecraft and Rockets, Vol. 13, No. 5, pp. 282-287.

${ }^{13}$ Kwakernaak, H., and Sivan, R., Linear Optimal Control Systems, John Wiley and Sons, New York, 1972.

${ }^{14}$ Mohler, R.R., Nonlinear Systems, Prentice Hall, Englewood Cliffs, New Jersey, 1991.

${ }^{15}$ Brak, S., "Top Level Design of Delfi-n3Xt Attitude Determination and Control Subsystem," Master's Thesis, Department of Space Systems Engineering, Delft University of Technology, Delft, The Netherlands, 2009. 